\title{
Early Postoperative Care After Cardiac Surgery
}

\author{
Paul M. H. J. Roekaerts ${ }^{1}$ and John H. Heijmans ${ }^{2}$ \\ ${ }^{1}$ Department of Intensive Care \\ ${ }^{2}$ Department of Anaesthesiology \\ University Hospital Maasticht, Maastricht \\ The Netherlands
}

\section{Introduction}

The early postoperative course for most patients after cardiac surgery is characterized by a typical pattern of pathophysiologic derangements that benefits form standardized care. Multimodal, multidisciplinary standardization of the care process has been shown to improve use of resources, efficiency, quality, safety and patient satisfaction.

The initial management in the postoperative care after routine cardiac surgery has fundamentally shifted during the past two decades towards a more efficient use of limited postoperative care facilities, early extubation and rapid discharge. The fast-track protocol became feasible after cardiac surgery due to improvements in perioperative anaesthesia management, new surgical techniques, better myocardial protection and cardiopulmonary bypass techniques and to better management of bleeding using point-of-care testing and new hemostatic drugs.

This chapter will briefly discuss the major pathophysiologic derangements and their management during the first 24 hours after surgery. It will than summarize the postoperative care to more specific procedures. Finally, the management of common postoperative complications will be discussed.

\section{Pathophysiology during the early postoperative period}

\section{Arrival in the intensive care unit}

Upon arrival in the ICU, an efficient transfer of care from operation room staff to ICU staff is mandated, while at the same time vital signs are to be maintained stable.

The initial goals in postoperative cardiac recovery are sufficient analgesia, normothermia, adequate oxygenation and ventilation, control of bleeding, restoration of intravascular volume, optimization of blood pressure and cardiac output to maintain organ perfusion and metabolic stabilization.

\section{Hypothermia}

Hypothermic cardiopulmonary bypass is usually terminated after the patient has rewarmed to a core body temperature of at least $36^{\circ} \mathrm{C}$. (1) However, patients usually arrive in the ICU with lower core temperatures. This drop in temperature from end of CPB until arrival in the ICU is due to the cool ambient temperatures in the operation room, poor peripheral perfusion and anesthesia-induced inhibition of normal themoregulation. Even patients 
operated under normothermic $\mathrm{CPB}$, have a tendency to significantly cool down before conclusion of surgery.

Hypothermia has many potential adverse effects. (2) It increases the systemic vascular resistance (SVR) which increases myocardial afterload and myocardial oxygen demand. This compensatory mechanism to provide core warming may contribute to slow warming of peripheral tissues. Drugs that provide vasodilation may improve peripheral perfusion. To prevent hypotension, warmed infusions should be administered concomitantly. Peripheral vasodilation augments heat loss, and core hypothermia may therefore persist.

Hypothermia also precipitates shivering, thereby increasing $\mathrm{CO} 2$ production and oxygen consumption, and predisposes to ventricular arrhythmias and coagulation cascade impairments. $(3,4)$

Therefore, warming should be hastened by forced-air warming blankets, heated humidifiers in the ventilator circuit and warmed infusion fluids. The use of other types of warming blankets or radiant heading hoods can also be considered. (5)

After cardiac surgery, patients may rapidly rewarm and occasionally overwarm to higher temperatures. This phenomenon is attributed to the resetting of the central thermoregulation system.

\section{Blood loss after cardiac surgery}

Careful hemostasis in the operation room is the cornerstone in reducing postoperative blood loss. However, bleeding can also be medical and determing the cause of bleeding is often difficult. Although the clinical situation must be individualized for each patient, bleeding in general should not exceed $400 \mathrm{~mL} / \mathrm{hr}$ during the first hour, $200 \mathrm{~mL} / \mathrm{hr}$ for each of the first 2 hours, or $100 \mathrm{~mL} / \mathrm{hr}$ over the first four hours. (6)

There are numerous medical causes for bleeding following cardiac surgery. Residual heparinization is common post cardiac surgery and usually occurs when insufficient protamine is used or heparined pump blood is transfused following CPB.

Platelet dysfunction is also common following cardiac surgery. The CPB circuit itself leads to contact activation and degranulation of platelets, resulting in their dysfunction. Fibrinolysis frequently occurs after $\mathrm{CPB}$, caused by activation of inflammatory or coagulation pathways.

Coagulation factors may decrease from activation and dilution in the $\mathrm{CPB}$ circuit.

There has been a dramatic increase in the iatrogenic use of heparin and newer antiplatelet, antithrombotic and thrombolytic drugs during (interventional) treatment of acute cornary syndromes. If revascularization surgery is warranted immediately after these treatments, the anticoagulant effect of these drugs is notable in the postoperative period.

Conventional coagulation tests are helpful to identify the coagulation abnormality contributing to the bleeding. Common laboratory testing includes $\mathrm{Hb}$, platelet count, aPTT, INR, and fibrinogen level. Thromboelastography is also commonly used and has been demonstrated to reduce transfusion requirements.

The most basis principles of the management of postoperative bleeding are:

1. Diagnose underlying medical cause by coagulation tests;

2. Rule out surgical bleeding;

3. Restore clotting parameters to normal by means of medications, transfusion of blood products or clotting factors, and restore normothermia;

4. Monitor for stability of clotting parameters. 
Most cardiac surgical centers use the antifibrinolytic lysine analogues, tranexaminic acid and aminocaproic acid, to reduce intraoperative bleeding. These drugs significantly reduce allogeneic blood transfusion after cardiac surgery.

Although rescue therapy with recombinant factor VII can be life-saving in massive bleeding after cardiac surgery, its safety has been questioned. A recent meta-analysis $(\mathrm{N}=4468$ from 35 studies) demonstrated that this therapy significantly increased the rate of arterial but not venous thromboembolic events. Given its cost and arterial thrombotic risk, it is likely that this hemostatic intervention will continue to be reserved for life-saving therapy of massive coagulopathy after cardiac surgery.(7)

\section{Blood transfusion management}

Although there are guidelines for blood transfusion in cardiac surgery, considerable variability has persisted in clinical practice. This variability also exists in anticoagulation and coagulation management.

A recent randomized controlled trial has already demonstrated that restrictive perioperative transfusion does not result in inferior clinical outcome after cardiac surgery. (8) Transfusion burden may in the future be interpreted as a quality indicator in cardiac surgery that must balance risks and benefits to achieve cost-effective optimal clinical outcomes. $(9,10)$

Perioperative transfusion algorithms for the administration of blood products, coagulation factors and pro-coagulant drugs should assist in preserving resources with improvement in patient safety. (11)

\section{Fluid resuscitation}

Cardiac surgery and CPB elicit a systemic inflammatory response which produces a capillary leak. Therefore, fluid resuscitation with cristalloids and/or colloids is necessary to offset the hemodynamic consequences of the capillary leak and the vasodilation that occurs from rewarming and vasodilating drugs. However, the maintenance of intravascular volume in the leakage phase occurs at the expense of expansion of the interstitial space. $(12,13)$

After the capillairy leak has ceased and hemodynamics have stabilized, diuretics are often used to eliminate the excessive salt and water administered during surgey and the early postoperative phase. This forced diuresis may beneficially affect pulmonary function and early successful extubation.

Several intraoperative measures that have been implemented throughout the years caused a reduction in the inflammatory response and may have contributed to the faster recovery times currently observed after cardiac surgery. The measures include the use of membrane oxygenation, centrifugal pumps, anti-fibrinolytic drugs and steroids, leukocyte filters and coated CPB tubings. $(14,15)$

\section{Perioperative cardiovascular dysfunction}

Adequacy of organ perfusion and tissue oxygenation is the primary goal of hemodynamic management in the postoperative cardiac surgical patient. Preload, afterload and contractility should therefore be maintained at their optimal level. This commonly requires atrial or atrioventricular pacing.

Approximately $20 \%$ of cardiac surgical patients develop cardiovascular dysfunction in the perioperative period, resulting in an inability to pump sufficient blood at normal end-diastolic pressures. There are three distinct clinical scenarios of cardiac impairment in the perioperative period of cardiac surgery - precardiotomy, failure to wean and postcardiotomy - differing from each other substantially concerning diagnosis, monitoring and management. 


\section{Precardiotomy heart failure}

Myocardial ischaemia is one of the most frequent causes of precardiotomy low output syndrome. The dysfunctional myocardium may not be irreversibly damaged and possibly only 'stunned' or 'hibernating'. Revascularization of the reversibly injured heart areas may result in improved cardiac performance. The first priority should therefore be prompt surgery avoiding further alterations in myocardial contractility, possibly by introducing an IABP preoperatively. However, inadequate myocardial protection during cardiac surgery may exacerbate ischaemic injury in some patients. Patients with longer standing previous poor preoperative cardiac function or with recently irreversibly injured ischaemic heart areas, will of course continue to have poor ventricular performance postoperatively.

Failure to wean

For the successful therapeutic approach of failure to wean, a correct diagnosis of the underlying cause is necessary. The heart failure may be procedure related or patient specific and includes inadequate myocardial protection, reperfusion injury, ischaemia, infarction, incomplete revascularization, metabolic, uncorrected pathology, mechanical issues, conduction issues, pulmonary hypertension and right ventricular failure.

Postcardiotomy heart failure

The priority is to preserve end organ function. Preload and heart rhytm should be optimized, and positive inotropic and/or vasopressor drugs are often used to maintain adequate cardiac output and blood pressure. Although this strategy will restore haemodynamics in most patients, mechanical circulatory support may be indicated.

\section{Monitoring and assessing volume status}

Heart failure cannot be ascertained unless the volume status is optimal. However, it is difficult to ascertain volume loading using single haemodynamic measures. Pressure estimates such as pulmonary capillary wedge pressure and central venous pressure are generally unreliable indicators of LV and RV preload. Uncoupling between PCWP and LVEDP frequently occurs as a consequence of elevated pulmonary vascular resistance, pulmonary venoconstriction, mitral stenosis and reduction in transmural cardiac compliance.

Volumetric estimates by echocardiography or transpulmonary thermal dilution techniques are more predictive of preload. In predicting fluid responsiveness in ICU patients, it is preferable to use more reliable dynamic indicators reflecting hypovolaemia, such as stroke volume variation, than static parameters. (16) Several devices are now being used to assess cardiac function based on pulse contour analysis of an arterial waveform. (17)

Echocardiography is of great value in the perioperative cardiac surgical setting. It not only is helpfull in assessing the optimal volume status, but may also immediately identify causes of cardiovascular failure, including valvular problems, cardiac tamponade, systolic anterior motion of the anterior mitral valve leaflet and pulmonary embolism. Echocardiography may differentiate between acute right, left and global heart failure as well as between systolic and diastolic dysfunction.

If there are echocardiographic signs of RV failure, a pulmonary artery catheter (PAC) preferably with continuous $\mathrm{SvO} 2$ measurement should be introduced. PACs can differentiate between pulmonary hypertension and RV ischaemia, which necessitates a reduction of RV afterload. PAC and TEE are complementary to each other for diagnosis and treatment of the cardiac surgical patient. Indications for the use of a PAC are, high risk and/or complex cardiac surgery, hemodynamic instability, low cardiac output syndrome, pulmonary hypertension, 
differentiating between severe right and left ventriclular dysfunction, vasodilation/vasoconstriction, hypovolemia. $\mathrm{SvO}_{2}$ in combination with lactate concentration was used postoperative as a goal-oriented hemodynamic therapy to improve outcome. $(18,19)$

\section{Risk stratification}

Risk stratification is increasingly used in open-heart surgery to help adjust resources to predicted outcome. According to all scoring systems major clinical risks include heart failure, unstable coronary syndromes, significant arrhythmias and severe valvular disease. The euroSCORE is mostly used to calculate operative risk, although updating its sensitivity is warranted. $(20,21)$

In addition to scoring systems, levels at hospital admission of B-type natriuretic peptide (BNP) and the amino-terminal fragment of pro-BNP (NT pro-BNP) are powerful predictors of outcome with regard to in-hospital mortality and rehospitalisation in heart failure patients. (22)

\section{Perioperative myocardial protection}

The ultimate goal of perioperative myocardial protection is to limit the extent and consequences of myocardial ischaemia-reperfusion injury. This injury is caused by free radical formation, calcium overload and impairment of the coronary vasculature. Protective measures include the use of free radical oxygen scavengers, inhibitors of the complement system and neutrophil activation, modulation of intracellular gradients and maintenance of sufficient myocardial high energy phosphate stores. Drugs affecting the complementinflammation pathways, adenosine modulators, cardioplegia solution adjuvants, $\mathrm{Na}^{+} / \mathrm{H}^{+}$ exchange inhibitors, $\mathrm{K}_{\mathrm{ATP}}$ channel openers such as volatile anaesthetics and levosimendan, and anti-apoptotic agents are all used for this purpose. $(23,24)$

\section{Pharmacologic support of myocardial dysfunction}

Pharmacological treatment of low cardiac output and reduced oxygen delivery to vital organs is often required in the perioperative cardiac surgical setting. Inadequate treatment may lead to multiple organ failure, one of the main causes of prolonged hospital stay, postoperative morbidity and mortality. Optimal use of inotropes and vasopressors is still controversial and needs further large multinational randomized controlled trials.

However, some recommendations can be made:

- Norepinephrine should be used in case of low blood pressure due to vasoplegia to maintain an adequate perfusion pressure. Preload should be assessed regularly to avoid hypovolemia under vasopressors.

- All catecholamines have positive inotropic and chronotropic effects. There is evidence that dobutamine better preserves the myocardial oxygen balance as compared to the other commonly used drugs. Dobutamine increases stroke volume and heart rate while PCWP is moderately decreased. If blood pressures are low, the combination dobutamine-norepinephrine is frequently used.

- Phosphodiesterase III inhibitors are potent vasodilators and cause less tachyarrhythmias as compared to dobutamine. They also have a more favourable effect on the myocardial oxygen balance as compared to the catecholamines.

- Levosimendan, a calcium sensitizer, has recently been introduced for the treatment of low cardiac output in the perioperative period with success. $(25,26,27,28)$

\section{Mechanical circulatory support}

The intra-aortic balloon pump (IABP) is recommended in the case of heart dysfunction with suspected coronary hypoperfusion. It's main mechanism of action is a reduction of afterload 
and diastolic coronary perfusion pressure. The IABP reduces heart work and myocardial oxygen consumption, favourably modifying the balance of oxygen supply/demand.

Extra-corporeal membrane oxygenation (ECMO) is increasingly used for temporary mechanical circulatory support. Advantages of the system include low cost, availability in all cardiac surgical centers and versatile use for cardiac, pulmonary and renal support. ECMO is used as a bridge to recovery, to transplantation, to long-term assist-device and to decision making.

Ventricular assist devices are used today as an established option for patients with end-stage heart failure to obtain a level of functionality that results in an acceptable quality of life for the patient.

\section{Cardiac arrhythmias}

\section{Temporary pacing}

Two temporary right atrial and two right ventricular epicardial pacing wire electrodes are usually placed at the conclusion of cardiac surgery. Atrial pacing wires can be used diagnostically to record atrial activity. These recordings, obtained simultaneously with standard limb leads, can distinguish among atrial and junctional arrhythmias and differentiate them from more life-threatening ventricular arrhythmias. (6)

The use of pacing is often required in the per- and postoperative period to increase heart rate. Atrial or AV pacing will nearly always demonstrate superior haemodynamics to ventricular pacing. Reentrant rhytms can be terminated by rapid pacing.

\section{Arrhythmias after cardiac surgery}

The development of cardiac arrhythmias following open-heart surgery is fairly common and related to altered impulse formation and conduction. An understanding of these mechanisms and the electrophysiologic effects of antiarrhythmic drugs provides a rational basis for the treatment of the different rhythm disturbances.

\section{Atrial fibrillation}

Despite various prophylactic measures, atrial fibrillation and flutter occur in about $35 \%$ of all cardiac surgical patients, most commonly on the second and third postoperative day. Etiologic factors include atrial distension, pericardial inflammation, enhanced sympathetic activity, surgical trauma and poor atrial preservation.

To prevent atrial fibrillation, B-blockers with or without class III ( Sotalol) antiarrhythmic properties, are commonly administered orally in the perioperative phase. Dual site atrial pacing and numerous other medications (amiodarone, magnesium sulphate, triodothyronine, digoxin, steroids, procainamide, verapamil, diltiazem) have all been reported to have some favourable effect on the incidence of atrial fibrillation after cardiac surgery.

Treatment consists of cardioversion in the haemodynamically unstable patient. For the stable patient, rate control and attempts to achieve conversion are usually initiated. Drugs used for rate control include calcium-channel blockers (diltiazem, verapamil), ß-blockers (esmolol, metoprolol), magnesium sufate and digoxin. For conversion to sinus rhythm, magnesium sulphate, IA medications (procainamide, quinidine), IC (propafenone) or III antiarrhythmics (Ibutilide, Amiodarone) are commonly used. $(29,30,31)$

\section{Ventilation management}

Pulmonary complications following cardiac surgery are common, even in patients with healthy lungs, and include diminished functional residual capacity (FRC) following general anaesthesia and muscle relaxants, reductions in vital capacity (VC) following median 
sternotomy and intrathoracic manipulation, atelactasis, increased intravascular lung water, and increased capillary leakage and extravascular lung water due to the inflammatory response to $\mathrm{CPB}$ and surgery. Multiple blood product transfusions and excessive fluid loading may further compromise lung functioning. Acute FRC reduction results in arterial hypoxemia due to ventilation-perfusion mismatch and shunting. In the early postoperative phase, restoration of FRC and maintenance of adequate gas exchange in the face of rising $\mathrm{VO}_{2}$ and $\mathrm{VCO}_{2}$ are the primary goals. This can be achieved by a lung-protective ventilation strategy with adequate levels of PEEP. $(32,33,34)$

For several decades, the medical care of the cardiac surgical patients in the perioperative setting consisted of high-dose opioid stress-free anaesthesia and prolonged mechanical ventilation in the ICU. In recent years, the concepts of Fast-Track Cardiac Anesthesia, Early Extubation and Short-Stay Intensive Care became the backbone of modern perioperative care. Indeed, several randomized trials have shown the safety of fast-tracking. $(35,36,37,38,39)$

Alterations in anaesthetic protocols using short-acting sedatives-hypnotics and analgesics, less invasive surgical and perfusion techniques, improved perioperative haemostasis management, fluid restriction, preservation of normothermia and reduction of the inflammatory response were all crucial steps in the development of fast-track cardiac surgery. As the number of elderly people is growing fast and cardiac surgery is now an accepted practice in these older patients, fast-tracking makes it possible to more efficiently use the limited facilities and resources. (40)

The debate regarding the optimal extubation time, the window of opportunity, is still ongoing. (41) There are several studies on outcome after extubation in the operation room, which show that it is feasible with good results. $(42,43)$ However, the nadir of ventricular function occurs about 4 hours following cardiopulmonary bypass. Also, the first few hours after cardiac surgery are characterized by periods of haemodynamic instability, temperature dysregulation, increased mediastinal blood loss and other homeostatic disturbances. Patients can rapidly deteriorate in this early postoperative phase and we believe that instabilities can be best anticipated and treated in an ICU setting in sedated and ventilated patients. The window of opportunity for extubation is therefore between 2 and 6 hours postoperatively.

Weaning strategies should be protocolized.(44) In table 1 is shown a nurse-driven weaning protocol which includes the criteria for the start of the weaning procedure, adequate breathing criteria and the extubation criteria. Only three steps in this protocol may mandate the consultation of the ward doctor.

After ventilatory weaning, the next step in the postoperative ICU management is the discharge of the patient to a step-down unit. This is usually accomplished within 8 hours after arrival in the ICU. Intensive Care discharge criteria are shown in Table 2.

\section{The postoperative (intensive) care unit}

The advent of Fast-Track Cardiac Anaesthesia and Short-Stay Intensive Care after Cardiac Surgery also started the discussion whether or not these patients should be treated in a conventional ICU setting. Can adequate and safe postoperative care be given to these patients in parallel "special-care units" such as a dedicated Cardiac Recovery Area (CRA). If a hospital has such a highly-equipped special care unit with a competent and qualified ICU doctor on the ward, adequate nurse-patient ratio and immediate access to ICU-OR facilities, then special care may be feasible. Several institutions reported safe and adequate care in these special units. $(45,46)$ However, in the early postoperative phase, the clinical condition of the patient may 


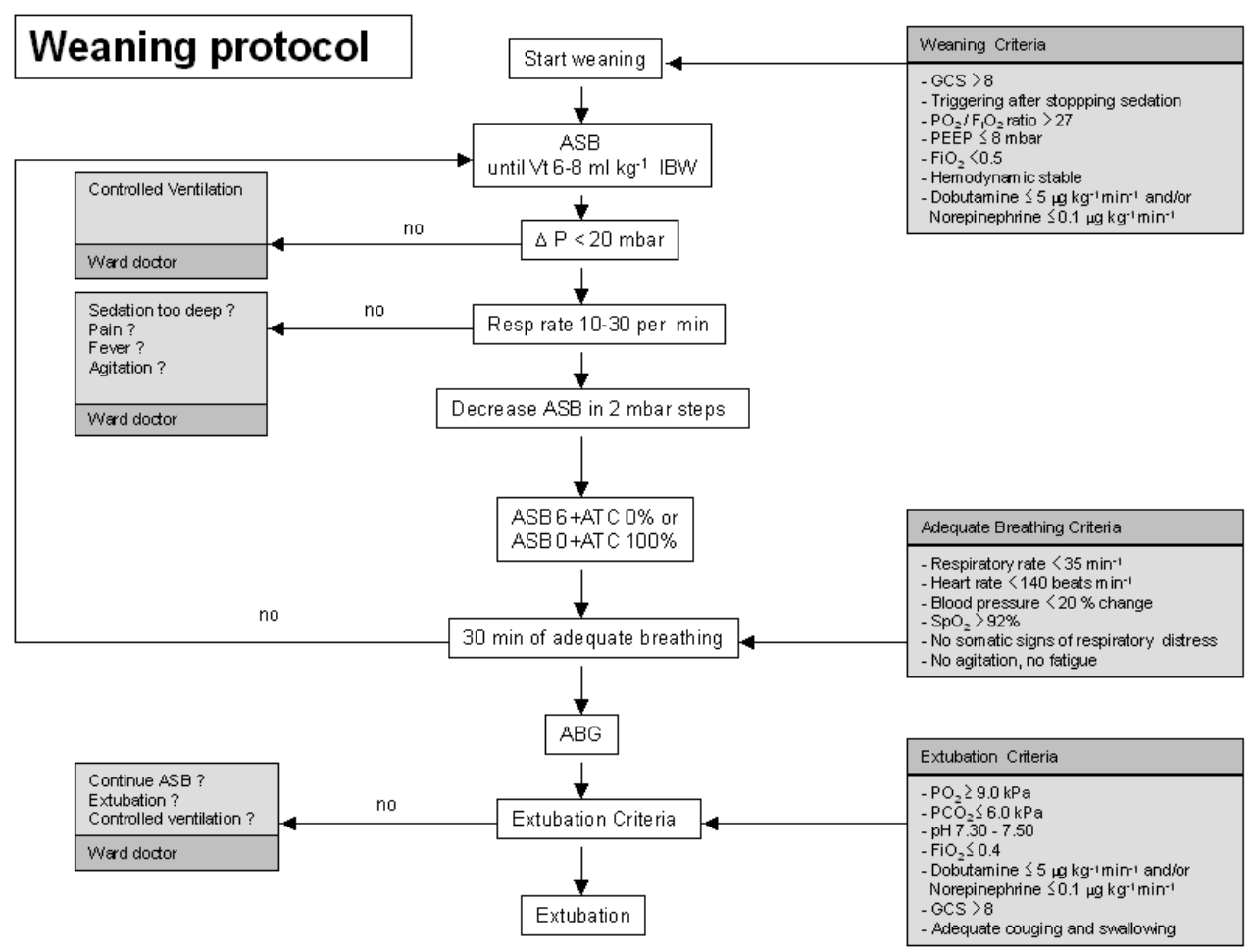

Table 1. Weaning protocol

\section{$\underline{\text { ICU Discharge Criteria }}$}

$\begin{array}{ll}\text { Pulmonary } & \text { Extubation }>30 \mathrm{~min} \\ \text { Oxygen }<5 \mathrm{~L} \mathrm{~min}-1 \text { nasally } \\ \text { Respiratory rate }>10 \mathrm{~min} \text { and }<25 \mathrm{~min} \\ \mathrm{PaO} 2>9 \mathrm{kPa} \text { and } \mathrm{PCO} 2<6.5 \mathrm{Kpa}\end{array}$

Cardiac No myocardial ischaemia or ongoing infarction

No haemodynamically significant dysrhythmias

Fluid balance $\quad$ Chest tube drainage $<100 \mathrm{~mL} \mathrm{hr}-1$

Diuresis $>0.5 \mathrm{~mL} \mathrm{Kg}-1 \mathrm{hr}-1$

Neurologic No signs/symptoms of major neurologic complications

Haemodynamic No iv vasoactive drugs

Except dobutamine $2 \mathrm{~g} \mathrm{Kg}-1 \mathrm{~min}-1$

No IABP

and/or nitroglycerine $0.5 \mathrm{~g} \mathrm{Kg}-1 \mathrm{~min}-1$

Cardiac Index $>2 \mathrm{~L}$ min $-1 \mathrm{~m}-2$

Table 2. ICU Discharge Criteria 
deteriorate extremely rapidly. Therefore, continuous adequate monitoring and maximal acute treatment or intervention should always be readily possible for these patients in the early postoperative period. We believe that currently in most hospitals, the ICU setting is the safest and best place to recover from cardiac surgery. In the integrated model, in which all patients are admitted to the ICU, the postoperative management such as nursing-topatient ratio is variable based on patient requirements. The goal is thus a postoperative unit that allows variable levels of monitoring and care based on patient need. In this model, discharge to a step-down unit as soon as possible after extubation and stabilization of vital parameters should be strived after for every single patient.

\section{Postoperative anticoagulation}

After coronary artery surgery, antiplatelet therapy has been shown to inhibit platelet deposition on vein grafts and may delay or attenuate the development of fibrointimal hyperplasia and atherosclerosis. Aspirin should therefore be started after CABG surgery and continued indefinitely because of its beneficial effects in patients with native coronary disease. (47)

After tissue aortic valve surgery, there is some evidence that short-term anticoagulation may reduce the incidence of thromboembolism. Therefore, anticoagulation is generally recommended for 3 months in younger patients or those with no contraindication for anticoagulation, and is then converted to aspirin. If anticoagulation is not used, aspirin is given. $(48,49)$

After mechanical aortic valve surgery, all patients should receive anticoagulation indefinitely to achieve an INR of 2.5 - 3.5 for tilting and bileaflet valves.

After mitral tissue valve or mitral ring implantation, anticoagulation should be given for 3 months to achieve an INR of $2.0-3.0$ and should then be converted to aspirin if the patient is in sinus rhythm. Anticoagulation should be continued indefinitely in patients with atrial fibrillation, an enlarged left atrium (> $50 \mathrm{~mm}$ in diameter), or a history of thromboembolism. After mechanical mitral valve insertion, anticoagulation is given to achieve an INR of 2.5 3.5. The addition of aspirin is safe and may further reduce the thromboembolic risk. $(50,51)$

\section{Echocardiography}

During the past few decades, the effect of perioperative transoesophageal echocardiography's (TEE) influence on perioperative cardiac surgical decision making has become increasingly more appreciated. Data from several clinical investigations have consistently implicated an important, clinically significant, and cost-effective role for TEE as a safe and valuable haemodynamic monitor in identifying high-risk patients, in assessing in the determination of the definitive surgical approach, and in providing a timely postcardiopulmonary bypass evaluation of the procedure, thereby allowing for the opportunity to immediately re-intervene or to at least triage patients appropriately. In addition, perioperative TEE has been instrumental in diagnosing cardiac and associated great vessel pathology and in identifying structural abnormalities, aortic disease, intracardiac masses, and pericardial disease. TEE is perhaps most useful for the perioperative evaluation of cardiac valvular disease, especially during surgical procedures involving the mitral valve. In the intra- and early postoperative period of cardiac surgery, an experienced echocardiographer remains an indispensable clinical team member. Increasing numbers of cardiac anaesthesiologists and intensivists are now being trained and certified as perioperative echocardiographers. $(52,53,54)$ 


\section{Analgesia and sedation}

An essential element of postoperative care is the provision of adequate analgesia and sedation. In the patient in whom delayed extubation is anticipated, the residual effects of anesthetics and midazolam in combination with a narcotic are generally accepted.

With the trend toward earlier extubation, short-acting narcotics and analgesics are administered during surgery. This requires early postoperative administration of shortacting medications for pain relief and sedation. We prefer to give low dose continuous infusions of morphine in combination with propofol in the ICU. This usually produces adequate sedation and pain relief without respiratory depression and allows for fairly early extubation.

\section{Management after specific cardiac surgical procedures}

\section{Coronary Artery Bypass Grafting}

Treatment of coronary artery disease can be medical or interventional. Catheterization procedures include balloon angioplasty, cardiac stenting and drug-eluding stents, which release drugs capable of preventing stenosis. Surgery includes CABG with the use of the cardiopulmonary bypass machine and $\mathrm{OPCAB}$ without the use of the $\mathrm{CPB}$ machine. OPCAB surgery may include sternotomy, thoracotomy (MIDCAB) or robotically assisted thoracotomy.

With increasing number of treatment options, it is crucial to establish for each patient which option is superior with regard to angina recurrence, graft patency, and long term survival with the least morbidity at the lowest costs. Several studies address this important issue. Analysis of individual patient data from ten randomized trials including 7812 patients concluded that long-term mortality after CABG and PCI in most patient subgroups with multivessel CAD is similar. (55) CABG versus PCI had lower mortality in diabetes and patients older than 65 years. The SYNTAX investigators concluded from their study that in low and intermediate risk patients with multivessel CAD, PCI and CABG have similar outcomes. In high risk patients with multivessel CAD, CABG is preferred. (56)

Although the COURAGE study, which provided optimal medical therapy (OMT) to all patients and demonstrated no incremental advantage of PCI on outcomes other than angina-related quality of life in stable $\mathrm{CAD}$, a recent analysis by Borden et al reported that among patients with stable CAD undergoing PCI, less than half were receiving OMT before PCI and approximately two-thirds were receiving OMT at discharge following PCI. $(57,58)$

A number of randomized controlled studies comparing OPCAB to on pump CABG have been completed. Although outcomes have been largely comparable, the evidence of benefits of OPCAB from these trials has not been as convincing as was first anticipated. A large adequately powered RCT of OPCAB versus on pump CABG in high risk patients is needed to determine whether this undeniably harder technique is here to stay. $(59,60,61)$

Aortic Valve Surgery

Aortic valve replacement surgery may be complicated by heart block because the conduction system lies adjacent to the base of the right coronary cusp. If AV pacing is necessary for more than 4 to 5 days, during which time edema or hemorrhage should subside, placement of a permanent DDD pacemaker is necessary because the conduction system then probably has been damaged by sutures or débridement. 
The hypertrophied, noncompliant left ventricle in aortic stenosis depends on adequate preload and on atrial contractions. Loss of sinus rhythm is associated with a $30 \%$ reduction in stroke volume and requires $\mathrm{AV}$ pacing.

In aortic regurgitation, the left ventricle is volume and pressure overloaded resulting in a dilated and often hypertrophied chamber. Aortic valve repair for aortic regurgitation is evolving into a standard of care. The systematic classification of aortic regurgitation based on leaflet mobility within the functional aortic annulus makes it possible to study outcomes of the specific interventions. $(62,63)$

A recent RCT showed that transcatheter aortic valve implantation (TAVI) is significantly superior to medical management of severe aortic stenosis in patients judged to be at excessive risk for conventional aortic valve replacement. (64) TAVI significantly reduced allcause 1-year mortality. Recent studies have documented rates of cerebral embolism of 70-80 $\%$. (65) Future trials should focus on interventions for stroke reduction after TAVI, including cerebral embolic protection. Techniques for reduction of embolic load may also improve renal dysfunction after TAVI. Although the short and medium term durability of the TAVI valve with preserved hemodynamic performance has been established, further studies are required to elucidate the long term effects. $(66,67,68)$ To this term, guidelines for standardized endpoints in TAVI trials have been published.(69)

Mitral valve surgery

Patients with chronic mitral stenosis often have pulmonary hypertension and usually are diuretic-dependent. They have a small left ventricular cavity with preserved LV function. Common postoperative problems are a low cardiac output syndrome associated with the small LV end-diastolic and end-systolic volumes, RV dysfunction and ventilatory failure due to the pulmonary hypertension, cachexia and fluid overload.

Due to the systolic unloading in patients with mitral valve regurgitation reducing LV wall stress, greater systolic wall stress is required after surgery to achieve adequate cardiac output. Therefore, the use of inotropic support and afterload reduction is often indicated.

In the postoperative period, cardiovascular management is often directed toward increasing filling pressures to above $15-20 \mathrm{mmHg}$, reduction of pulmonary hypertension and improvement of RV and LV failure. Guiding hemodynamic support with the use of a pulmonary artery catheter may be very helpfull. When atrial fibrillation has been present for more than 1 year or when LA dimension exceeds $50 \mathrm{~mm}$, it is very unlikey to maintain sinus rhythm in the postoperative period. AV pacing is often possible after surgery and may improve cardiac performance. (6)

\section{Diseases of the thoracic aorta}

Multidisciplinary guidelines for thoracic aortic diseases were published in 2010. (70) We will highlight some concerns that concern the perioperative setting.

Ascending aortic dilatation should be carefully measured in patients with a bicuspid aortic valve presenting for surgery. Earlier surgical intervention is warranted to avoid rupture or dissection.

In aortic arch aneurysm surgery, hybrid repair has emerged as low risk aortic repair in highrisk patients. Type I repairs have adequate proximal and distal landing zones: after offpump anastomosis of the brachiocephalic vessels to the ascending aorta, an endovascular stent is deployed for complete arch repair. Type II repairs have adequate distal landing zone but insufficient ascending aorta to serve as a proximal stent landing zone: after ascending aortic replacement with aortic arch debranching, an endovascular stent is deployed for 
complete arch repair with the ascending aortic graft serving as proximal landing zone. Type III repairs have inadequate proximal and distal landing zones: after total arch replacement with a distal elephant trunk, the descending thoracic aortic repair is completed by endovascular stentingwith the elephant trunk serving as the proximal landing zone.

Concerning aortic dissection, the Penn classification of a type A dissection integrates type of clinical presentation with dissection extent to stratify perioperative outcome and facilitate decision-making about the type of surgical repair. (71)

The American Heart Association recently published a position paper on the integrated management of decending thoracic aortic disease that complements the recent guidelines from the Society of Thoracic surgeons. (72) These guidelines together summarize the paradigm shift in the management of descending thoracic aortic pathologies due to endovascular therapies. In Stanford type B aortic dissection, the conservative management of refractory pain and hypertension is associated with significant short-term mortality. Therefore, although a survival advantage has not been demonstrated yet, endovascular intervention of these type B dissections is now more often applied.

Depending on the type of organ protection applied during aortic surgery (deep hypothermic circulatory arrest, selective perfusion of brain and kidneys) coagulopathies and neurologic deficit may occur. Brain damage may be due to ischemia or embolisation and paraplegia may result from crossclamping of the descending aorta. Careful neurologic evaluation before and after surgery are important.

Also, the hypotensive regimen used in the early postoperative period must reduce systolic blood pressure and the force of cardiac contraction. The most common regimens include the use of beta-blockers.

\section{Management of complications}

\section{Atrial fibrillation}

Atrial fibrillation following cardiac surgery is common and occurs in up to $35 \%$ of patients. While the cause of AF is not completely understood, it is associated with an increase in mortality, stroke, and prolonged hospital stay. AF has been discussed in Section I of this chapter.

\section{Low cardiac output syndrome}

A low cardiac output state may result from decreased left ventricular preload (hypovolemia, cardiac tamponade, vasoplegia), decreased contractility (myocardial stunning, ischemia or infarction related to poor intraoperative myocardial protection, incomplete myocardial revascularization, anastomotic stenosis, or coronary artery spasm), arrhythmias, increased afterload or diastolic dysfunction.

Transoesophageal echocardiography can help define whether a low cardiac output state is related to left ventricular systolic or diastolic dysfunction, right ventricular dysfunction or cardiac tamponade. The management of low cardiac output has been discussed in Section I of this chapter.

Right ventricular dysfunction produces inadequate filling of the left heart resulting in a low cardiac output state. It may be attributable to poor myocardial protection, prolonged ischemic times, coronary embolism, hypotension, RV pressure overload (pulmonary disease, ARDS, pulmonary embolism) or acute pulmonary hypertension due to vasoactive substances, LV dysfunction, protamine or hypoxia and acidosis. 
Right coronary artery disease, right ventricular infarction and pulmonary hypertension associated with mitral/aortic disease predispose to RV failure after cardiac surgery.

PAC's and TEE are very helpful in assessing the status of the RV function. In the absence of LV dysfunction, a high RA/PCWP pressure ratio is suggestive for RV dysfunction. The goals of treatment are to optimize RV preload, maintain systemic perfusion pressure, improve RV contractility, and reduce RV afterload by reducing pulmonary vascular resistance. (27)

Diastolic dysfunction, defined as increased resistance to filling of one or both cardiac chambers, is a common finding after cardiac surgery, especially after cardioplegic arrest. Echocardiography has greatly improved the knowledge of diastole by showing the real-time activities in the heart, as related to filling pressures, shape and relaxation. Failure of the RV can contribute to left-sided diastolic dysfunction by increasing cardiac pressures, which causes decreased relaxation of the myocardium yielding decreased myocardial distensibility. Factors responsible for increased chamber stiffness include fibrosis, cellular disarray, and hypertrophy. Factors responsible for decreased relaxation include asynchrony, abnormal loading, ischemia, abnormal calcium ion flux and hypertrophy. Note that ventricular hypertrophy affects both stiffness and relaxation, increasing the risk of diastolic dysfunction. (73)

\section{Cardiac tamponade}

Cardiac tamponade is primarily the result of impaired filling of one or more of the cardiac chambers and leads to low cardiac output. Adrenergic and endocrine mechanisms are activated resulting in tachycardia and vasoconstriction.

The diagnosis of cardiac tamponade depends on a high degree of suspicion. Tamponade after cardiac surgery is different from a medical tamponade due to compressing fluid within an intact pericardium. In the setting of cardiac surgery, the pericardial space is often left open and in open communication with one or both the pleural spaces, and the compressing blood is at least in part clotted and able to cause localized compression of the heart. Serious suspicion for tamponade should rise in patients with deteriorating haemodynamics or gradually increasing requirements for inotropic drugs. The classic signs of elevated CVP or equalization of CVP and PAOP are often absent. Cardiac tamponade is difficult to distinguish from biventricular failure. A useful clue may be the pronounced respiratory variation of blood pressure in association with high filling pressures and low cardiac output. TEE may be helpful in diagnosing cardiac tamponade. Echolucent crescents between the RV wall and the pericardium or the posterior LV wall are discernible. A classic sign is diastolic collapse of the right atrium or RV.

A rule of thumb in the acute management of cardiac tamponade is to keep the patient Full, Fast and Thight. Full, the delivery of volume expansion in order to achieve an adequate preload. Fast, using pacing or medication to increase the heart rate to maintain cardiac output since the strokevolume is compromised. Tight, applying vasopressor therapy to increase preload, maintain bloodpressure and coronary perfusion pressure.

The definitive treatment of tamponade is surgical exploration with evacuation of hematoma.

\section{Renal insufficiency}

No clear definition exists as to what constitutes renal impairment or failure following $\mathrm{CPB}$. Renal failure requiring dialysis is infrequent following $\mathrm{CPB}$, although reductions in creatinine clearance are more frequent. There are several risk factors for postoperative renal 
failure, including postoperative low cardiac output, repeat cardiac surgery, valve surgery, age greater than 65 , and diabetes.

The primary cause may be prerenal (low pressure, low output, ACE, NSAID's), renal (Acute Kidney Injury) from ischaemic insult or interstitial drug-related nephritis or postrenal.

Management of these patients consists of supportive treatment ensuring adequate cardiac ouput, perfusion pressure and volume status and of determining the primary cause, and then directing specific treatment as necessary such as discontinuing the offending drug.

If patients do require dialysis, continuous dialysis may be better than intermittent dialysis. $(6,74,75,76)$

\section{Impediments to weaning and extubation}

The most important factors limiting weaning and extubation in the early postoperative period after cardiac surgery include:

1. neurologic dysfunction

- agitation, restlessness and disorientation may occur after discontinuation of the sedative medication. The ethiology of this syndrome is multifactorial and includes patient characteristics, perioperative psychotropic drugs used for anaesthesia, pain relief and sedation, and brain ischaemia and inflammation. Initial management consists of reassurance and orientation of the patient and control of pain with opioids. Resedation for a period or the use of haloperidol may be usefull until the patient is oriented and tranquil.

- diaphragmatic paralysis may complicate cardiac surgery, especially after reoperations, due to surgical lesion of the phrenic nerve in fibrotic pericardial tissue. The phrenic nerve can also be injured or transected during dissection of the internal mammary arteries or during mobilization of the heart in redo surgery. Transient diaphragmatic paralysis can also occur secondary to cold injury by the cold cardioplegic solutions to the phrenic nerve. (6)

The diagnosis of diaphragmatic paralysis should be considered whenever a patient fails to wean from mechanical ventilation and can be documented by observing paradoxical movement of the diaphragm during inspiration.

2. unstable haemodynamics

Postoperative cardiac surgical patients with unstable haemodynamics and/or low cardiac output syndromes may not well tolerate the extra work of breathing associated with weaning. Weaning is difficult and may further deteriorate the already compromised myocardium.

Weaning affects cardiac output due to changes in pulmonary vascular resistance. Increased pulmonary vascular resistance (PVR) leads to septal shifts and reduced efficiency of biventricular function. It is therefore better to keep the patient sedated on full ventilator support until the cardiac problem is resolved. (77)

3. fluid overload

Cardiac surgery and $\mathrm{CPB}$ result in a systemic inflammatory response syndrome which produces a capillary leak. The duration and severity of this syndrome include factors related to the patient characteristics, severity of the surgical trauma, administration of blood products and CPB management. The use of heparin-coated tubings, membrane oxygenator, centrifugal pumps, steroids and leukocyte filters may reduce the SIRS. The capillary leak syndrome is usually most predominant the first 6 to 8 hours after the termination of $\mathrm{CPB}$. 
During this period, fluid resuscitation is necessary to offset the capillary leak syndrome and the vasodilation secondary to medications and rewarming. Crystalloid and colloid infusions are used to maintain intravascular volume, although this usually occurs at the expense of expansion of the interstitial space. After the capillary leak has ceased and haemodynamics are stable, diuretics contribute to a faster recovery from surgery.

Succesfull early extubation is compromised by fluid overload. Optimal monitoring and adequate measures should therefore be taken in the operation room and in the intensive care to minimize the positive fluid balance while maintaining adequate tissue perfusion. (78)

\section{Central nervous system dysfunction}

Neurologic complications are dreaded sequelae of cardiac surgery. Notwithstanding a progressive decrease in cardiac surgical mortality over the past decades, the incidence of postoperative neurological complications remains relatively unchanged.

\section{Focal neurologic complications}

Focal neurologic events complicate approximately $2 \%$ of cardiac procedures requiring $\mathrm{CPB}$, but may increase as more patients with advanced age and diffuse vascular disease undergo cardiac surgery. Focal deficits may include hemiparesis or hemiplegia, aphasia, dysarthria, hand incoordination and visual field deficits.

Preoperative risk factors include increasing age (risk of up to $10 \%$ in patients older than age 75), pre-existing cerebrovascular disease, hypertension, peripheral vascular disease, and poor LV function. Intraoperative and postoperative risk factors include: ascending aortic atherosclerosis and calcification, LV mural thrombus, complex surgery and prolonged bypass and haemodynamic instabilities.

The mechanisms for neurologic injury include some combination of cerebral embolism, hypoperfusion, and inflammation; associated vascular disease and cerebral autoregulatory dysfunction make the brain more susceptible to injury. Particulate embolism due to atherosclerotic plaque, blood thrombus embolus, and air and platelet-fibrin debris is the most common cause of stroke. Cerebral hypoperfusion may be the result of systemic hypotension or impaired regional cerebral blood flow. Although cerebral autoregulation should protect the brain during $\mathrm{CPB}$, hypothermia, blood gas regulation, diabetes and preexisting hypertension may affect the adequacy of cerebral autoregulation. $(6,79,80,81)$

In the prevention of focal neurologic complications, preoperative evaluation for extracranial carotid disease should be considered in any patient with neurologic symptoms. Symptomatic carotid disease warrants carotid endarterectomy(CE) prior or at the time of cardiac surgery. Asymptomatic carotid disease in the presence of a carotid bruit should be evaluated by non-invasive testing. There is a trend toward performance of combined CABG$\mathrm{CE}$ in these patient groups. (82)

Intraoperative echocardiograhic scanning of the ascending aorta to identify atherosclerosis might alter cannulation sites and clamping - and manipulation techniques of this diseased aorta. Techniques to avoid embolic load include the use of membrane oxygenators, arterial filters in the $\mathrm{CPB}$ circuit, meticulous débridement and irrigation of valves, removal of LV thrombi and of air after intracardiac procedures.

In general, in patients with hypertension or intracranial vascular disease, blood pressure during CPB should be maintained at a higher level.

In the treatment of embolic stroke, heparin is recommended when there is no evidence of intracranial hemorrhage on the CT scan. Heparin prevents propagation of intracardiac 
thrombus and improves cerebral microcirculation, but is of unclear benefit in preventing further atheroembolism from dislodged plaque.

Encephalopathy

Encephalopathy is fairly common after cardiac surgery and is usually manifested by disorientation and confusion, lethargy or agiation, and paranoia and hallucinations.

The ethiology of this syndrome is multifactorial. It may be related to brain inflammation, cerebral hypoperfusion or microemboli from the $\mathrm{CPB}$ circuit. Other factors include patient characteristics, hypoxia, metabolic disturbaces, perioperative psychotropic drugs used for anaesthesia, pain relief and sedation, and drug or alcohol withdrawal.

Initial management consists of reassurance and orientation of the patient and control of pain with opioids. Resedation for a period or the use of haloperidol may be usefull until the patient is oriented and tranquil. The encephalopathy has a fluctuating course but is usually transient. $(79,81)$

\section{References}

[1] Ho K.M. and J.A. Tan. Benefitis and Risks of Maintaining Normothermia During Cardiopulmonary Bypass in Adlut Cardiac Surgery: A Systemic Review. Cardiovasc Ther 2009; 29: 260-279.

[2] Sessler D.I.. Complications and Treatment of Mild Hypothermia. Anesthesiology 2001; 95: 531-543.

[3] Crossley A.W.. Peri-operative Shivering. Anaesthesia 1992; 47:193-195.

[4] Rajagopalan S., E. Mascha, J. Na, and D.I. Sessler. The Effectis of Mild Perioperative Hypothermia on Blood Loss and Transfusion Requirement. Anesthesiology 2008;108: 71-77.

[5] Frank S.M., L.A. Fleisher, M.J. Breslow, M.S. Higgins, K.F. Olson, S. Kelly, and C. Beattie. Perioperative Maintenance of Normothermia Reduces the Incidence of Morbid Cardiac Events. A Randomized Clinical Trial. JAMA 1997; 277:1127-1134.

[6] Bojar R.M.. Manual of Perioperative Care in Adult Cardiac Surgery. 2011 WileyBlackwell. ISBN: 978-1-4443-3143-1.

[7] Levi M., J.H. Levy, H.F. Andersen, and D. Truloff. Safety of Recombinant Activated Factor VII in Randomized Clinical Trials. N Engl J Med 2010; 363: 1791-1800.

[8] Hajjar L.A., JL. Vincent, F.R.B.G. Galas, R..E. Nakamura, C.M.P. Silva, M.H. Santos, J. Fukushima, R.K. Filho, D.B. Sierra, N.H. Lopes, T. Mauad, A.C. Roquim, M.R. Sundin, W.C. Leao, J.P. Almeida, P.M. Pomerantzeff, L.O. Dallan, F.B. Jatene, N.A.G. Stolf, and J.O.C. Auler Jr.. Transfusion Requirements After Cardiac surgery. The TRACS Randomized Controlled Trial. JAMA 2010; 304: 1559-1567.

[9] Shander A.S. and L.T. Goodnough. Blood Transfusion as a Quality Indicator in Cardiac Surgery. JAMA 2010; 304: 1610-1611.

[10] Varghese R., and M.L. Myers. Blood Conservation in Cardiac Surgery: Let's Get Restrictive. Semin Thoracic Surg 2010; 22: 121-126.

[11] 2011 Update to The Society of Thoracic Surgeons and the Society of Cardiovascular Anesthesiologists Blood Conservation Clinical Practice Guidelines. The Society of Thoracic Surgeons Blood Conservation Guidelines Task Force: Ferraris V.A., J.R. Brown, G.J. Despotis, J.W. Hammon, B. Reece, S.P. Saha, H.K. Song, and E.R. Clough. The Society of Cardiovascular Anesthesiologists Special Task Force on 
Blood Transfusion: Shore-Lesserson L.J., L.T. Goodnough, D. Mazer, A. Shander, M. Stafford-Smith, and J. Waters. The International Consortium for Evidence Based Perfusion: Baker R.A., T.A. Dicksinson, D.J. FitzGerald, D.S. Likosky, and K.G. Shann. Ann Thorac Surg 2011; 91: 944-982.

[12] Vincent JL. Fluid Resuscitation: Colloids vs Crystalloids. Acta Clin Bel, Suppl, 2007; 2: 408-411.

[13] Bundgaard-Nielsen M., N.H. Secher, and H. Kehlet. 'Liberal' vs. 'Restrictive' Perioperative Fluid Therapy - A Critical Assessment of the Evidence. Acta Anaesthesiol Scand 2009; 53: 843-851.

[14] Dieleman J.M., J. van Paassen, D. van Dijk, M.S. Arbous, C.J. Kalkman, J.P. Vandenbroucke, G.J. van der Heijden, and O.M. Dekkers. Prophylactic Corticosteroids for Cardiopulmonary Bypass in Adults (Review). Cochrane Database of Systematic Reviews 2011, Issue 5. Art. No.: CD005566.

[15] Henry D.A., P.A. Carless, A.J. Moxey, D. O'Connell, B.J. Stokes, D.A. Fergusson, and K. Ker. Anti-fibrinolytic use for Minimising Perioperative Allogeneic Blood Transfusion (Review). Cochrane Database of Systematic Reviews 2011, Issue 3. Art. No.: CD001886.

[16] Renner J., J. Scholz, and B. Bein. Monitoring Fluid Therapy. B Prac Res Clin Anaesth 2009; 23: 159-171.

[17] Heijmans J.H., Y.M. Ganushchak, M.S. Theunissen, J.G. Maessen, and P.M.H.J. Roekaerts. Predictors of Cardiac Responsiveness to Fluid Therapy after Cardiac Surgery. Acta Anaesth Belg 2010; 16:151-158.

[18] Carl M., A. Alms, J. Braun, A. Dongas, J. Erb, A. Goetz, M. Goepfert, W. Gogarten, J. Grosse, A.R. Heller, M. Heringlake, M. Kastrup, A. Kroener, S.A. Loer, G. Marggraf, A. Markewitz, D. Reuter, D.V. Schmitt, U. Schirmer, C. Wiesenack, B. Zwissler, and C. Spies. S3 Guidelines for Intensive Care in Cardiac Surgery Patients: Hemodynamic Monitoring and Cardiocirculary System. GMS Ger Med Sci. 2010; 8:Doc 12.

[19] Polonen P., E. Ruokonen, M. Hippelainen, M. Poyhonen, and J. Takala. A Prospective, Randomized Study of Goal-Oriented Hemodynamic Therapy in Cardiac Surgical Patients. Anesth Analg 2000; 90: 1052-1059.

[20] Heijmans J.H., J.G. Maessen, and P.M.H.J. Roekaerts. Risk Stratification for Adverse Outcome in Cardiac Surgery. European J Anesth $2003 ; 20$ : 515-527.

[21] Nashef S.A., F. Roques, P. Michel, E. Gauducheau, S. Lemeshow, R. Salamon. European System for Cardiac Operative Risk Evaluation (EuroSCORE). Eur J Cardiothorac Surg 1999; 16: 9-13.

[22] Lainchbury J.G., R.W. Troughton, K.M. Strangman, C.M. Frampton, A. Pilbrow, T.G. Yandle, A.K. Hamid, M.G. Nicholls, and A.M. Richards. N-Termed Pro-B-type Natriuretic Peptide-Guided Treatment for Chronic Heart Failure. J Am Coll Cardiol 2010; 55: 53-60.

[23] Warren O.J., A.J. Smith, C. Alexiou, P.L.B. Rogers, N. Jawad, C. Vincent, A.W. Darzi, and T. Athanasiou. The Inflammatory Response to Cardiopulmonary Bypass: Part 1 - Mechanisms of Pathogenesis. J Cardiothorac Vasc Anesth 2009; 23: 223-231.

[24] Warren O.J., A.L. Watret, K.L. de Wit, C. Alexiou, C. Vincent, A.W. Darzi, and T. Athanasiou. The Inflammatory Response to Cardiopulmonary Bypass: Part 2 - 
Antiinflammatory Therapeutic Strategies. J Cardiothorac Vasc Anesth 2009; 23: 384393.

[25] Duranteau J., P. Sitbon, JL. Teboul, E. Vicaut, N. Anguel, C. Richard and K. Samii. Effects of Epinephrine, Norepinephrine, or the Combination of Norepinephrine and Dobutamine on Gastric Mucosa in Septic Shock. Crit Care Med 1999; 27: 893900.

[26] Mebazaa A., A.A. Pitsis, A. Rudiger, W. Toller, D. Longrois, SE. Ricksten, I. Bobek, S. De Hert, G. Wieselthaler, U. Schirmer, L.K. von Segesser, M. Sander, D. Poldermans, M. Ranucci, P. C. J. Karpati, P. Wouters, M. Seeberger, E. R. Schmid, W. Weder, and F. Follath. Clinical Review: Practical Recommendations on the Management of Perioperative Heart Failure in Cardiac Surgery. Critical Care 2010; 14: 201. Epub 2010 April 28.

[27] Mebazaa A., P. Karpati, E. Renaud, and L. Algotsson. Acute Right Ventricle Failure from Pathophysiology to New Treatments. Int Care Med 2004; 30: 185-196.

[28] Lahtinen P., O. Pitkanen, P. Polonen, A. Turpeinen, V. Kiviniemi, and A. Uusaro. Levosimendan Reduces Heart Failure after Cardiac Surgery - A Prospective, Randomized, Placebo - Controlled Trial. Crit Care Med 2011;Jun 9 (Epub ahead of print).

[29] Bradley D., L.L. Creswell, C.W. Hogue Jr., A.E. Epstein, E.N. Prystowsky, and E.G. Daoud. American College of Chest Physicians Guidelines for the Prevention and Management of Postoperative Atrial Fibrillation After Cardiac Surgery. Chest 2005; 128, Suppl: 39S-47S.

[30] Koniari I., E. Apostolakis, C. Rogkakou, N.G. Baikoussis, and D. Dougenis. Pharmacologic Prophylaxis for Arial Fibrillation Following Cardiac Surgery: A Systemic Review. J Cardiothorac Surg 2010; 5: 121.

[31] Mitchell L.B., and the CCS Atrial Fibrillation Guidelines Committee. Canadian Cardiovascular Society Atrial Fibrillation Guidelines 2010: Prevention and Treatment of Atrial Fibrillation Following Cardiac Surgery. Can J Cardiol 2011; 27: 91-97.

[32] Apostolakis E.E., E.N. Koletsis, N.G. Baikoussis, S.N. Siminelakis, and G.S. Papadopoulos. Strategies to Prevent Intraoperative Lung Injury During Cardiopulmonary Bypass. J Cardiothorac surg 2010; 5: 1.

[33] Kilpatrick B., and P. Singer. Lung Protective Strategies in Anaesthesia. Brit J Anaesthesia 2010;105: i108/i116.

[34] The Acute Respiratory Distress Syndrome Network. Ventilation with Lower Tidal Volumes as Compared with Traditional Tidal Volumes for Acute Lung Injury and the Acute Respiratory Distress Syndrome. N Eng J Med 2000; 342: 1301-1308.

[35] Cheng D.C.H., J. Karski, C. Peniston, B. Asokumar, G. Raveendran, J. Carroll, H. Nierenberg, S. Roger, D. Mickle, J. Tong, J. Zelovitsky, T. David, and A. Sandler. Morbidity Outcome in Early versus Conventional Tracheal Extubation After Coronary Artery Bypass Grafting: A Prospective Randomized Controlled Trial. J Thorac Cardiovasc Surg 1996;112: 755-764.

[36] Hawkes C.A., S. Dhileepan, and D. Foxcroft. Ealry Extubation for Adult Cardiac Surgical Patients. Cochrane Database of Systematic Reviews. 2003;(4): CD003587.

[37] Myles P.S., and D.Mcllroy. Fast-Track Cardiac Anesthesia: Choise of Anesthetic Agent and Techniques. Sem Cardiothorac Vasc Anesth 2005: 9: 5-16. 
[38] Mastrigt van G.A.P.G., J. Heijmans, J.L. Severens, E.J. Fransen, P. Roekaerts, G. Voss, and J.G. Maessen. Short-Stay Intensive Care After coronary Artery Bypass Surgery: Randomized Clinical Trial on Safety and Cost-Effectiveness. Crit Care Med 2006; 34: 65-75.

[39] Svircevic V., A.P. Nierich, K.G.M. Moons, G.J. Brandon Bravo Bruinsma, C.J. Kalkman, and D. van Dijk. Fast-Track Anesthesia and Cardiac Surgery: a Retrospective Cohort Study of 7989 Patients. Anesth Analg 2009;108: 727-733.

[40] Paone G., R.S.D. Higgins, S.L. Havestad, and N.A. Silverman. Does Age Limit the Effectiveness of Clinical Pathways After Coronary Artery Bypass Graft Surgery? Circulation 1998; 98: II-41 - II-45.

[41] Higgins T.L. Safety Issues Regarding Early Extubation After Coronary Artery Bypass Surgery. J Cardiothorac Vasc Anesth 1995; 9: 24-29.

[42] Lee T.W.R., and E. Jacobsohn. Pro: Tracheal Extubation Should Occur Routinely in the Operating Room After Cardiac Surgery. J Cardiothorac Vasc Anesth 2000; 14: 603610.

[43] Djaiani G.N., M. Ali, L. Heinrich, J. Bruce, J. Carroll, J. Karski, R.J. Cusimano, and D.C. Cheng. Ultra-Fast-Track Anesthetic Techniques Facilitates Operating Room Extubation in Patients Undergoing Off-pump Coronary Revascularisation Surgery. J Cardiothorac Vasc Anesth 2001;15: 152-157.

[44] Van Mastrigt G.A.P.G., J.G. Maessen, J. Heijmans, J.L. Severens, and M.H. Prins. Does Fast-track Treatment Lead to a Decrease of Intensive Care Unit and Hospital Length of Stay in Coronary Artery Bypass Patients ? A Meta-regression of Randomized Clinical Trials. Crit Care Med 2006; 34: 1624-1634.

[45] Flynn M., S. Reddy, W. Shepherd, C. Holmes, D. Armstrong, C. Lunn, K. Khan, and S. Kendall. Fast-tracking Revised: Routine Cardiac Surgical Patients Need Minimal Intensive Care. Eur J Cardiothorac Surg 2004; 25: 116-122, 2004.

[46] Ender J., M.A. Borger, M. Scholz, AK. Funkat, N. Anwar, M. Sommer, F.W. Mohr, and J.F. Fassl. Cardiac Surgery Fast-track Treatment in a Postanesthetic Care Unit. Sixmonth Results of the Leipzig Fast-track Concept. Anesthesiology 2008; 109: 61-68.

[47] Zimmerman N., E. Gams, and T. Hohlfeld. Aspirin in Coronary Artery Bypass Surgery: New Aspects of and Alternatives for an Old Antithrombotic Agent. Review. Eur J Cardiothorac Surg 2008; 34: 93-108.

[48] Brueck M., W. Kramer, P. Vogt, N. Steinert, P. Roth, G. Gorlach, M. Schonburg, and M.C. Heidt. Antiplatelet Therapy Early After Bioprosthetic Aortic Valve Replacement is Unnecessary in Patients Without Thromboembolick Risk Factors. Eur J Cardiothorac Surg 2007; 32: 108-112.

[49] Colli A., JP. Verhoye, R. Heijmen, J.T. Strauch, J.A.J. Hyde, D. Pagano, M. Antunes, H. Koertke, S.K. Ohri, D.H.L. Bail, P. Leprince, B.H.M. Van Straten, T. Gherli, on behalf of ACTION Registry Invetigators. Antithrombotic Therapy After Bioprosthetic Aortic Valve Replacement: ACTION Registry Survey Results. Eur J Cardiothorac Surg 2008; 33: 531-536.

[50] Kulik A., F.D. Rubens, P.S. Wells, C. Kearon, T.G. Mesana, J. van Berkom, and BK. Lam. Early Postoperative Anticoagulation After Mechanical Valv Replacement: A systematic Review. Ann Thrac Surg 2006; 81: 770-781.

[51] Dunning J., M. Versteegh, A. Fabbri, A. Pavie, P. Kolh, U. Lockowandt, S.A.M. Nashef, on behalf of the EACTS Audit and Guidelines Committee. Guideline on 
Antiiplatelet and Anticoagulation Management in Cardiac Surgery. Euro J Cardiothorac Surg 2008; 34: 73-92.

[52] Couture P., A.Y. Denault, S. McKenty, D. Boudreault, F. Plante, R. Perron, D. Babin, L. Normandin, and N. Poirier. Impact of Routine use of Intraoperative Transesophageal Echocardiography during Cardiac Surgery. Can J Anesth 2000; 47: 20-26.

[53] Fox J., K. Glas, M. Swaminathan, and S. Shernan. The Impact of Intraoperative Echocardiography on Clinical Outcome Following Adult Cardiac surgery. Sem Cardiothorac Vasc Anesth 2005; 9: 25-40.

[54] Shernan S.K.. Perioperative Transesophageal Echocardiographic Evaluation of the Native Mitral Valve. Crit Care Med 2007; 35: 8 (suppl): S372-S383.

[55] Hlatky M.A., D.B. Boothroyd, D.M. Bravata, E. Boersma, J. Booth, M.M. Brooks, D. Carrie, T.C. Clayton, N. Danchin, M. Flather, C.W. Hamm, W.A. Hueb, J. Kahler, S.F. Kelsey, S.B. King, A.S. Kosinski, N. Lopes, K.M. McDonald, A. Rodriguez, P. Serruys, U. Sigwart, R.H. Stables, D.K. Owens, and S.J. Pocock. Coronary Artery Bypass Surgery Compared With Percutaneous Coronary Interventions for Multivessel Disease: A Collaborative Analysis of Individual Patient Data from Ten Randomized Trials. Lancet 2009; 373: 1190-1197.

[56] Kappetein A.P., T.E. Feldman, M.J. Mack, MC. Morice, D.R. Holmes, E. Stahle, K.D. Dawkins, F.W. Mohr, P.W. Serruys, and A. Colombo. Comparison of Coronary Bypass Surgery with Drug-eluting Stenting for the Treatment of Left Main and/or Three-vessel Disease: 3-year Follow-up of the SYNTAX trial. Eur Heart J 2011; doi:10.1093/eurheartj/ehr213

[57] Boden W.E., R.A. O'Rourke, K.K. Teo, P.M. Hartigan, D.J. Maron, W.J. Kostuk, M. Knudtson, M. Dada, P. Casperson, C.L. Harris, B.R. Chaitman, L. Shaw, G. Gosselin, S. Nawaz, L.M. Title, G. Gau, A.S. Blaustein, D.C. Booth, E.R. Bates, J.A. Spertus, D.S. Berman, J. Mancini, and W.S. Weintraub for the COURAGE Trial Research Group. N Engl J Med 2007; 356: 1503-1516.

[58] Borden W.B., R.F. Redberg, A.I. Mushlin, D. Dai, L.A. Kaltenbach, and J.A. Spertus. Patterns and Intensity of Medical Therapy in Patients Undergoing Percutaneous Coronary Intervention. JAMA 2011; 305: 1882-1889.

[59] Khan N.E., A. De Souza, R. Mister, M. Flather, J. Clague, S. Davies, P. Collins, D. Wang, U. Sigward, and J. Pepper. A Randomized Comparison of Off-pump and On-pump Multivessel Coronary-artery Bypass Surgery. N Engl J Med 2004; 350: 21-28.

[60] Louagie Y.A.G., J. Jamart, and A.Gruslin. Do Coronary Bypass Graft Flows Differ Between On-Pump and off-Pump Operations ? Ann thorac Surg 2005; 79: 20042012.

[61] Shroyer A.L., F.L. Grover, B. Hattler, J.F. Collins, G.O. McDonald, E. Kozora, J.C. Lucke, J.H. Baltz, and D. Novitzky for the Veterans Affairs Randomized On/Off Bypass (ROOBY) Study Group. N Engl J Med 2009; 361: 1827-1837.

[62] Augoustites J.G.T., W.Y. Szeto, and J.E. Bavaria. Advances in Aortic Valve Repair; Focus on Functional Approach, Clinical Outcomes, and Central Role of Echocardiography. Expert Review. J Cardiothorac Vasc Anesth 2010; 24: 1016-1020.

[63] Boodhwani M., L. de Kerckhove, D. Glineur, J. Rubay, JL. Vanoverschelde, P. Noirhomme, and G. El Khoury. Repair of Regurgitant Bicuspid Aortic Valves: A Sytematic Approch. J Thorac Cardiovasc Surg 2010; 140: 276-284. 
[64] Leon M.B., G.R. Smith, M. Mack, C. Miller, J.W. Moses, L.G.Svensson, M. Tuzcu, J.G. Webb, G.P. Fontana, R.R. Makkar, D.L. Brown, P.C. Block, R.A. Guyton, AD. Pichard, J.E. Bavaria, H.C. Herrmann, P.S. Douglas, J.L. Petersen, J.J. Akin, W.N. Anderson, D. Wang, and S. Pocock for the PARTNER Trial Investigators. Transcatheter Aortic-valve Implantation for Aortic Stenosis in Patients who Cannot Undergo Surgery. N Engl J Med 2010; 363: 1597-1607.

[65] Ghanem A., A. Muller, C.P. Nahle, J. Kocurek, N. Werner, C. Hammerstingl, H.H. Schild, J.O. Schwab, F. Mellert, R. Fimmers, G. Nickenig, and D. Thomas. Risk and Rate of Cerebral Embolism After Transfemoral Aortic Valve Implantation. J Am Coll Cardiol 2010; 55: 427-432.

[66] Gotzmann M., W. Bojara, M. Lindstaedt, A. Ewers, L. Bosche, A. Germing, T. Lawo, M. Bechtel, A. Laczkovics, and A. Mugge. One-Year of Transcatheter Aortic Valve Implantation in Severe Symptomatic Aortic Valve Stenosis. Am J Cardiol 2011; 107: 1687-1692.

[67] Kodali S.K. W.W. O'Neill, J.W. Moses, M. Williams, C.R. Smith, M. Tuzcu, L.G. Svensson, S. Kapadia, G. Hanzel, A.J. Kirtane, and M.B. Leon. Early and Late (OneYear) Outcomes Following Transcatheter Aortic Vlave Implantation in Patients With Severe Aortic Stenosis (from the United States REVIVAL Trila). Am J Cardiol 2011; 107: 1058-1064.

[68] Gurvitch R., D.A. Wood, E.L. Tay, J. Leipsic, J. Ye, S.V. Lichtenstein, C.R. Thompson, R.G. Carere, N. Wijesinghe, F. Nietlispach, R.H. Boone, S. Lauck, A. Cheung, and J.G. Webb. Transcatheter Aortic Valve Implantation. Durability of Clinical and Hemodynamic Outcomes Beyond 3 Years in a Large Patient Cohort. Circulation 2010; 122: 1319-1327.

[69] Leon M.B., N. Piazza, E. Nikolsky, E.H. Blackstone, D.E. Cutlip, A.P. Kappetein, M.W. Krucoff, M. Mack, R. Mehran, C. Miller, MA. Morel, J. Petersen, J.J. Popma, J.J.M. Takkenberg, A. Vahanianm, GA. Van Es, P. Vranckx, J.G. Webb, S. Windecker and P.W. Serruys. Standardized Endpoint Definitions for Transcatheter Aortic Valve Implantation Clinical Trial: A Consensus Report from th Valve Academic research Consortium. Eur Heart J 2011; 32: 205-217.

[70] Hiratzka L.F., G.L. Bakris, J.A. Beckman, R.M. Bersin, V.F. Carr, D.E. Casey Jr, K.A. Eagle, L.K. Hermann, E.M. Isselbacher, E.A. Kazerooni, N.T. Kouchoukos, B.W. Lytle, D.M. Milewicz, D.L. Reich, S. Sen, J.A. Shin, L.G. Svensson, and D.M. Williams. ACCF/AHA/AATS/ACR/ASA/SCA/SIR/STS/SVM Guidelines for the Diagnosis and Management of Patients with Thoracic Aortic Disease. J Am Coll Cardiol 2010; 55: 27-129.

[71] Augustides J.G.T., W.Y. Szeto, N.D. Desai, A. Pochettino, A.T. Cheung, J.S. Savino, and J.E. Bavaria. Classification of Acute Type A Dissection: Focus on Clinical Presentation and Extent. Review. Eur J Cardiothorac Surg 2011; 39: 519-522.

[72] Coady M.A., J.S. Ikonomidis, A.T. Cheung, A.H. Matsumoto, M.D. Dake, E.L. Chaikof, R.P. Cambria, C.T. Mora-Mangano, T.M. Sundt and F.W. Sellk; On behalf of the American Heart Association Council on Cardiovascular Surgery and Anesthesia and Council on Peripheral Vascular Disease. Surgical Management of Descending Thoracic Aortic Disease: Open and Endovascular Approaches: A Scientific Statement From the American Heart Association. Circulation 2010 ;121: 2780-2804. 
[73] Bernard F., A. Denault, D. Babin, C. Goyer, P. Couture, A. Couturier, and J. Buithieu. Diastolic Dysfunction is Predictive of Difficult Weaning from Cardiopulmonary Bypass. Anesth Analg 2001; 92: 291-298.

[74] Landoni G., T. Bove, M. Crivellari, D. Poli, O. Fochi, C. Marchetti, A. Romano, G. Marino, and A. Zangrillo. Acute Renal Failure After Isolated CABG Surgery : Six Years of Experience. Minerva Anesthesiol 2007; 73: 559-563.

[75] Bove T., M.G. Calabro, G. Landoni, G. Aletti, G. Marino, G. Crescenzi, C. Rosica, and A. Zangrillo.The Incidence and Risk of Acute Renal Failure after Cardiac Surgery. J Cardiothorac Vasc Anesth 2004; 18: 442-445.

[76] Schwann N.M., J. C. Horrow, M.D. Strong III, D. Chachad, A. Guerraty and A.S. Wechsler. Does Off-Pump Coronary Artery Bypass Reduce the Incidence of Clinical Evident Renal Dysfunction after Multivessel Myocardial Revascularisation ? Anesth Analg 2004; 99: 959-964.

[77] Andre A.C.St., and A. DelRossi. Hemodynamic Management of Patients in the First 24 Hours After Cardiac Surgery. Crit Care Med 2005; 33; 202-2093.

[78] Pradeep A., S. Rajagopalam, H.K. Kolli, N. Patel, R. Venuto, J. Lohr, and N.D. Nader. High Volumes of Intravenous Fluid During Cardiac surgery are Associated with Increased Mortality. HSR Proceedings in Intensive Care and Cardiovascular Anesthesia 2010: 2: 287-296.

[79] McKhann G.M., M.A. Grega, L.M. Borowicz, W.A. Baumgartner, and O.A. Selnes. Stroke and Encephalopathy After Cardiac Surgery. An Update. Stroke 2006; 37: 562-571.

[80] Van Eijk M.M.J., and A.J.C. Slooter. Delirium in Intensive Care Unit Patients. Sem Cardiothrac Vasc Anesth 2010; 14: 141-147.

[81] Mehta Y., and R. Singh. Cognitive Dysfunction After Cardiac Surgery. Review. J Alzheim Dis 2010; 22: S115-S120.

[82] Gopaldas R.R., D. Chu, T.K. Dao, J. Huh, S.A. LeMaire, P. Lin, J.S. Coselli, and F.G. Bakaeen. Staged Versus Synchronous Carotid Endarterectomy and Coronary Artery Bypass Grafting: Analysis of 10-Year Nationwide Outcomes. Ann Thorac Surg 2011; 91: 1323-1329. 


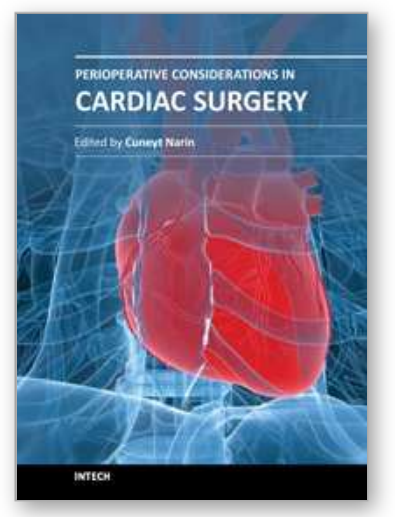

\author{
Perioperative Considerations in Cardiac Surgery \\ Edited by Prof. Cuneyt Narin
}

ISBN 978-953-51-0147-5

Hard cover, 378 pages

Publisher InTech

Published online 29, February, 2012

Published in print edition February, 2012

This book considers mainly the current perioperative care, as well as progresses in new cardiac surgery technologies. Perioperative strategies and new technologies in the field of cardiac surgery will continue to contribute to improvements in postoperative outcomes and enable the cardiac surgical society to optimize surgical procedures. This book should prove to be a useful reference for trainees, senior surgeons and nurses in cardiac surgery, as well as anesthesiologists, perfusionists, and all the related health care workers who are involved in taking care of patients with heart disease which require surgical therapy. I hope these internationally cumulative and diligent efforts will provide patients undergoing cardiac surgery with meticulous perioperative care methods.

\title{
How to reference
}

In order to correctly reference this scholarly work, feel free to copy and paste the following:

Paul M. H. J. Roekaerts and John H. Heijmans (2012). Early Postoperative Care After Cardiac Surgery, Perioperative Considerations in Cardiac Surgery, Prof. Cuneyt Narin (Ed.), ISBN: 978-953-51-0147-5, InTech, Available from: http://www.intechopen.com/books/perioperative-considerations-in-cardiac-surgery/-earlypostoperative-care-after-cardiac-surgery-

\section{INTECH}

open science | open minds

\section{InTech Europe}

University Campus STeP Ri

Slavka Krautzeka 83/A

51000 Rijeka, Croatia

Phone: +385 (51) 770447

Fax: +385 (51) 686166

www.intechopen.com

\section{InTech China}

Unit 405, Office Block, Hotel Equatorial Shanghai

No.65, Yan An Road (West), Shanghai, 200040, China

中国上海市延安西路65号上海国际贵都大饭店办公楼 405 单元

Phone: +86-21-62489820

Fax: +86-21-62489821 
(C) 2012 The Author(s). Licensee IntechOpen. This is an open access article distributed under the terms of the Creative Commons Attribution 3.0 License, which permits unrestricted use, distribution, and reproduction in any medium, provided the original work is properly cited. 